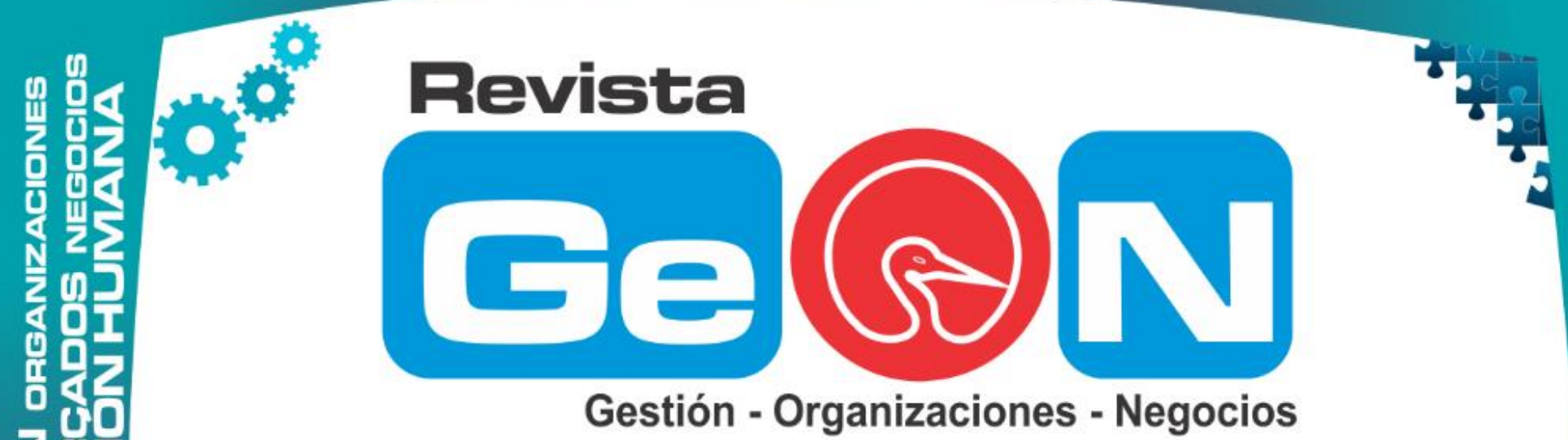

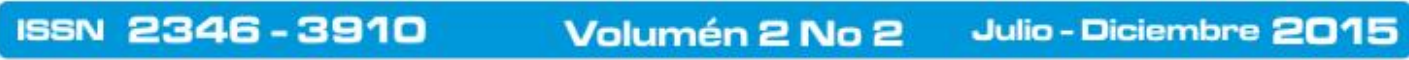

Revista Electrónica de la Facultad de Ciencias Económicas de la Universidad de los Llanos

1. 3 II

440

VILLAVICENCIO - COLOMBIA

$>2$

64

2

2 平品

70

ব)

展

o

ii III

0 U

ใด

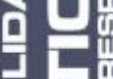

居的

임

Iiin in

照

只

造

iil 2

if in

4 吕

2

400

付 iI
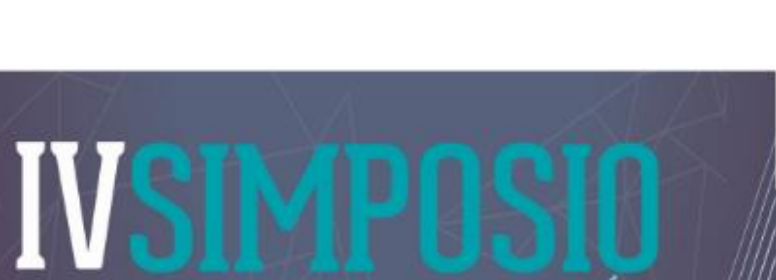

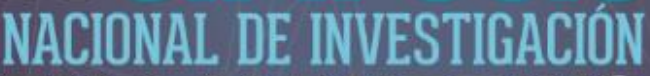

EN MARKETING
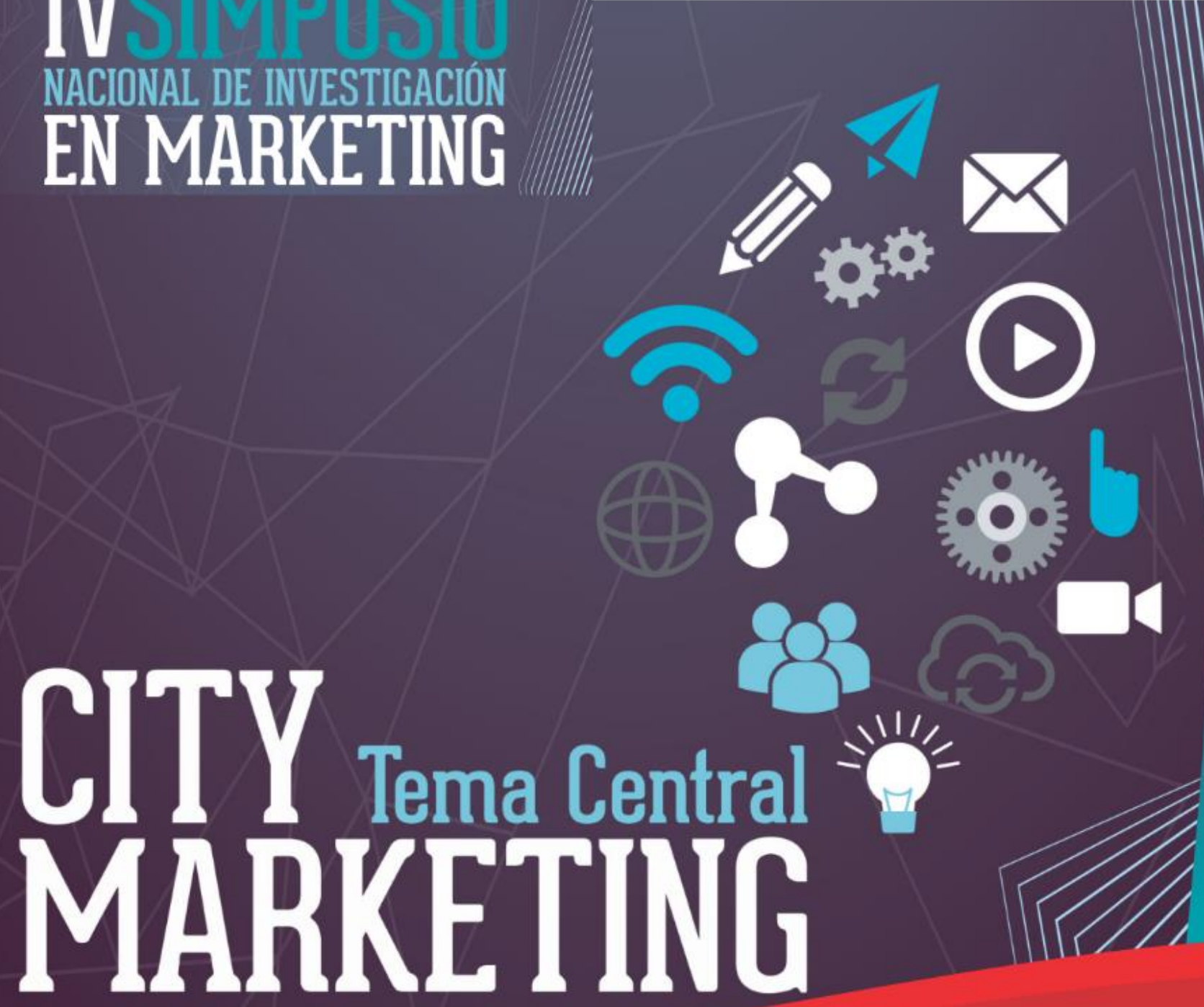

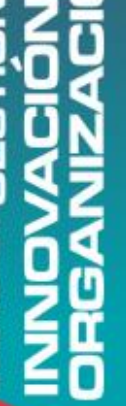
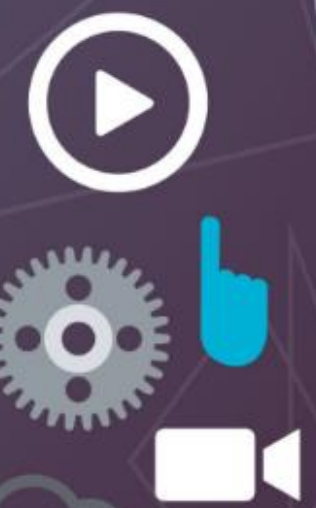


\section{Dinámicas de endomarketing en el restaurante de comidas rápidas FRISBY S.A.- Valentina Alzate - Natalia Sosa - Laura Gutierrez}

Valentina Alzate Salazar

Estudiante Pregrado en Mercadeo y Publicidad

Semillero de investigación Nómadas Fundacíón

Universitaria del Área Andina

valzate4@estudiantes.areandina.edu.co,

@valeealzate

Natalia Andrea Sosa Rey,

Estudiante Pregrado en Mercadeo y Publicidad

Semillero de investigación Nómadas Fundacíón

Universitaria del Área Andina

nsosa4@estudiantes.areandina.edu.co

Laura Patricia Gutiérrez Escobar

Estudiante Pregrado en Mercadeo y Publicidad

Semillero de investigación Nómadas Fundacíón

Universitaria del Área Andina

1gutierrez47@estudiantes.areandina.edu.co

@ laupge

\section{Resumen}

La investigación es realizada en el restaurante de comidas rápidas FRISBY S.A de la ciudad de Pereira durante el año 2015, para conocer sus dinámicas de endomarketing y la construcción de marca. Va dirigida a los colaboradores de los restaurantes FRISBY S.A de Pereira de forma cualitativa.

El endomarketing ha sido estudiado desde hace más de 25 años; Berry es una de las mayores referencias y un pionero del endomarketing; pues, en 1981 fue el primero en llamar a los empleados clientes internos. Este enfoque surgió por la necesidad de mejorar la gestión de personas y la atención a los clientes. El tratamiento empírico incentivó diversas interpretaciones y conceptualizaciones sobre el tema; como consecuencia surgieron distintas aplicaciones en la forma de desarrollo de metodologías con algunos puntos en común como la satisfacción del empleado y el cliente.
El endomarketing, es un conjunto de estrategias que se han empezado a implementar en un gran número de empresas alrededor del mundo. Estas estrategias están orientadas a "vender" la empresa a los propios empleados que la conforman y tengan sentido de pertenencia.

Los recursos humanos son una de las mayores bases para una organización y la obtención de una ventaja competitiva aplicada en uno de los modelos de marketing interno como lo es el modelo de Berry. De esta dinámica, se genera la importancia del análisis de la gestión de recursos. Por ello; en el restaurante de comidas rápidas pereirano, FRISBY S.A, se analiza la evolución, desarrollo y dinámicas del marketing interno.

\section{Palabras clave}

Endomarketing; marketing interno; servicio al cliente; comunicación organizacional; empowerment.

\begin{abstract}
The research is conducted in the fast food restaurant FRISBY SA City Pereira during the year 2015 to meet the dynamics of endomarketing and brand building. It is intended for employees of restaurants FRISBY SA de Pereira qualitatively.

The endomarketing has been studied for over 25 years; Berry is one of the major references and a pioneer of endomarketing; So in 1981 he was the first to call internal clients to employees. This approach arose from the need to improve people management and customer service. Empirical treatment stimulated different interpretations and conceptualizations on the subject; as a result different applications emerged in the form of development methodologies with some points in common as employee satisfaction and customer.
\end{abstract}


The endomarketing, is a set of strategies that have begun to implement a large number of companies around the world. These strategies are intended to "sell" the company to the employees that form and make sense of belonging.

Human resources are one of the largest bases for an organization and gaining a competitive advantage applied to one of the internal marketing models such as the model of Berry. In this dynamic, the importance of analyzing the management of resources is generated. Thus; Pereira, in fast food restaurant, FRISBY SA, evolution, development and marketing of the internal dynamics is analyzed.

\section{Keywords}

Internal marketing; internal marketing; customer service; organizational communication; empowerment.

\section{Introducción}

Todo el que hace parte laboral de una empresa merece un trato ejemplar que permita transmitir emociones positivas con las que el cliente externo se sienta seguro de elegir del servicio que se le ofrece. Teniendo en cuenta que el talento humano es el pilar fundamental de las compañías, y que de su cuidado permanente se derivan colaboradores comprometidos, motivados y entusiasmados con el cumplimiento de los deberes, objetivos y metas empresariales; especialistas en el ramo han determinado cuán importante e incluso necesario, es que las organizaciones, sin importar su tamaño o la naturaleza de su negocio, realicen inversiones a corto, mediano y largo plazo para promover el desarrollo y estabilidad de sus empleados que a su vez contribuye a la construcción de marca.

La comunicación organizacional es la pieza clave, la "polea" que mantiene unidos los piñones en torno a un objetivo en común, haciendo que la máquina funcione en la dirección adecuada, hacia una meta.

\section{Desarrollo}

\section{Objetivo general}

Comprender las prácticas de marketing interno los restaurantes de comidas rápidas FRISBY S.A en Pereira.

\section{Objetivos específicos}

Describir el proceso de marketing interno en el restaurante de comidas rápidas FRISBY S.A.

Identificar las actividades de marketing interno que se aplican en cada departamento.

Describir el enfoque de marketing interno aplicado en FRISBY S.A.

Identificar el énfasis que se le hace al empleado según el cargo que ocupa en la organización.

\section{Endomarketing}

El marketing interno, o también llamado endomarketing, es un concepto antiguo que empezó a ser tenido en cuenta en los años 80 . Berry es una de las mayores referencias para este período, pues fue el primero que, en 1981, llamó a los empleados como clientes internos. "The people who buy goods and services in the role of consumer, and the people who buy jobs in the role of employee, are the same people". (Berry, 1981, p. 34).

El marketing interno busca que el personal se sienta identificado con la organización, con su filosofía, sus valores y este motivado mejorando así su fidelidad con la empresa. El eje central son sus colaboradores. Esta actividad es necesaria porque la satisfacción de los clientes depende de que el personal mantenga una actitud abierta, decidida, voluntaria y espontánea para dar un excelente servicio en el que se alineen los clientes internos con los externos.

El Marketing Interno se plantea como una estrategia para mejorar la calidad de vida de los empleados, fortaleciendo su pertenencia a la empresa, generando procesos de comunicación 
efectivos, incrementando la colaboración y el trabajo en equipo para lograr el grado de satisfacción laboral que pueda transformar a las organizaciones. Por tanto, el uso de la mercadotecnia interna, es absolutamente imprescindible, su no aplicación supondrá una causa más que incidirá en la desmotivación general de las plantillas y, como consecuencia, en la pérdida de los mejores profesionales que emigrarán a contextos laborales más atractivos (Barranco 2009), lo que causa gastos de capacitación, y esto determina en volver empezar con una nueva persona para desarrollarse, quitando tiempo valioso para la organización.

El marketing interno consiste en diversas actividades comunicativas para promocionar los valores de la marca, imagen corporativa e identidad corporativa de la empresa entre sus propios empleados. Es un aspecto muy importante del employer branding, Los expertos en recursos humanos definen esta nueva forma de atraer y retener capital humano como "el desarrollo de una marca como empleadora", es decir, la generación de la identidad y reputación de una organización como el lugar ideal para trabajar. El mensaje se dirige no sólo a la gente que ya labora ahí [lo cual es visto como una excelente comunicación interna], sino también a clientes, inversionistas y posibles nuevos colaboradores en las empresas.

Siguiendo esta línea de pensamiento Goffman (1994) manifiesta: (...) en el acto comunicativo se produce una interacción entre varios individuos, interacción que se da en el orden de lo simbólico que construye las diversas instancias de las redes sociales. Cuando se concibe la comunicación como actividad social, se coloca un mecanismo de orden superior por encima de la comunicación (inter)individual. Cada acto de transmisión de mensaje se integra a una matriz mucho más vasta. Esta matriz, que recibe el tan vapuleado nombre de comunicación social, constituye el conjunto de los códigos y las reglas que hacen posible y mantienen en la regularidad y la previsibilidad las interacciones y las relaciones entre los miembros de una misma cultura. Así concebida, la comunicación en la sociedad es pues permanente: no se sostiene en la acción de un individuo, aquella que remite linealmente a un mensaje emitido y/o decodificado; más claramente, permite que la acción individual se inserte en una continuidad.

El individuo es visto como un "actor social", como un participante de una entidad que lo subsume. Las actividades comunicativas son actividades de control, de confirmación, de "integración", donde la redundancia juega un papel importante (Goffman, 1994, p.46).

\section{Modelos de marketing interno}

Todos los modelos llevan a la satisfacción del cliente externo por medio de la satisfacción del cliente interno.

\section{Modelo de marketing interno Berry}

En 1991, Berry y Parasuranam, proponen un modelo de marketing interno compuesto por 7 puntos de acción. Cada uno de los puntos tiene una aplicación directa en cualquier área de la gestión empresarial; estrategias dirigidas a los empleados de forma colectiva, puntual y por departamentos de gestión:

El empeño por atraer talento. Ofrecer una visión sencilla que se comunique frecuentemente, con pasión y que sirva para mantener emocionalmente a los colaboradores de la organización.

Tener preparados a los colaboradores por medio de un entrenamiento continuo.

Fomentar el buen clima laboral por medio del trabajo en equipo donde se compartan los objetivos.

Libertad para el trabajo: Libertad en la toma de decisiones junto con la necesidad de que hayan reglas para el buen funcionamiento.

Sistemas claros de evaluación.

Conocimiento de las necesidades de los clientes internos. Contar con un personal 
proactivo, buena actitud y aptitud para desempeñar un determinado cargo. A medida que la empresa crezca debe haber un entrenamiento y un empoderamiento del cargo por parte del trabajador con la intención de mejorar.

Aún en 1991, Berry y Parasuraman presentaban la propuesta de practicar el marketing interno antes del marketing externo, especialmente para la prestación del servicio al cliente, donde el servidor (empleado) puede ser comprado antes del servicio.

\section{Modelo de Marketing Interno de Christian Grönroos}

Grönroos defiende la satisfacción del cliente externo a través de los empleados y reclama mayor atención y conciencia de ellos entre los gestores de la organización. El marketing interno es una estrategia de la dirección, en donde se desarrolla entre los empleados un interés por el cliente. Todas las empresas tienen un mercado interno del cual preocuparse; si esto no se hace de forma adecuada se peligra la acción del cliente final.

El marketing interno, aplicado a la gestión de los recursos humanos, contempla a los empleados de una organización como un mercado, al que hay que analizar, segmentar y ofrecer un producto atractivo - consistente no solo en un puesto de trabajo y un salario- que satisfaga sus necesidades y consiga su mayor rendimiento e integración con los objetivos de la empresa. (Gronroos, 1984, p.179).

\section{Modelo de Rafiq y Ahmed}

Dunmore, Ahmed y Rafiq: Según Dunmore (2003) y Ahmed y Rafiq (2002), el concepto de Marketing Interno es reciente. Los estudios empezaron por la preocupación de una mejor gestión de personas y una mejor atención a los clientes.

Rafiq y Ahmed (2002), dicen que el concepto, empezó a ser tenido en cuenta en los años 80. En 1976 Berry, Hensen y Burke lo referencian cuando se relaciona por primera vez con la calidad del servicio.

En los años siguientes el tópico fue mencionado incialmente en la literatura de marketing de servicios, luego en la literatura de gestión de servicios $\mathrm{y}$, posteriormente, en la literatura del marketing industrial, conforme lo menciona Grönroos (1990).

\section{Modelo de Lings}

Este modelo dice que la calidad que tenga el marketing interno, se verá reflejada en el producto final que se le entregara al consumidor y que la motivación de los empleados debe de ser correspondiente a las estrategias que se empleen para satisfacer al consumidor. Por lo tanto en una organización se debe de tener en cuenta la forma en la que se aplica la comunicación con el cliente interno, para así poder reflejar la satisfacción que tienen los empleados en el producto final que se va a entregar (Lings, 1999).

\section{Modelo de Allen y Meyer}

Meyer y Allen citados por (Arias, 2001) dicen que el compromiso organizacional está dividido en tres componentes: afectivo, de continuidad y normativo.

Compromiso afectivo: (deseo) se refiere a los lazos emocionales que las personas forjan con la organización, refleja el apego emocional al percibir la satisfacción de necesidades (especialmente las psicológicas) y expectativas, disfrutan de su permanencia en la organización. Los trabajadores con este tipo de compromiso se sienten orgullosos de pertenecer a la organización.

Compromiso de continuación: (necesidad) señala el reconocimiento de la persona, con respecto a los costos (financieros, físicos, psicológicos) y las pocas oportunidades de encontrar otro empleo, si decidiera renunciar a la organización. Es decir, el trabajador se siente vinculado a la institución porque ha invertido 
tiempo, dinero y esfuerzo y dejarla implicaría perderlo todo; así como también percibe que sus oportunidades fuera de la empresa se ven reducidas, se incrementa su apego con la empresa.

Compromiso normativo: (deber) es aquel que encuentra la creencia en la lealtad a la organización, en un sentido moral, de alguna manera como pago, quizá por recibir ciertas prestaciones; por ejemplo cuando la institución cubre la colegiatura de la capacitación; se crea un sentido de reciprocidad con la organización. En este tipo de compromiso se desarrolla un fuerte sentimiento de permanecer en la institución, como efecto de experimentar una sensación de deuda hacia la organización por haberle dado una oportunidad o recompensa que fue valorada por el trabajador.

El compromiso organizacional es el grado en el que un empleado se identifica con su trabajo, participa activamente en él y considera que su desempeño laboral es importante para su propia valía. Los empleados que tienen un nivel alto de participación en el trabajo se identifican fuertemente con el tipo de trabajo que realizan y se preocupan realmente por él. (Robbins, 2005, p. 346)

\section{Conceptos}

\section{Comunicación interna}

La comunicación interna es una parte de la comunicación de la empresa que va dirigida a los empleados como clientes internos; esta comunicación empieza a surgir dado que las compañías ven la necesidad de motivar a sus empleados ya que esa motivación se va a ver reflejada en la interacción cliente-empleado.

Según García (1998), La gestión de la comunicación interna se enfoca en todas las acciones y metodologías aplicativas en donde los encargados de la comunicación planifican todos los recursos posibles que permita mejor desarrollo en la organización.
Se debe tener en cuenta que al realizar un plan de comunicación en una organización se debe tener claro que es lo que se pretende hacer ya que en muchas empresas se puede asumir que a mayor comunicación habrá una mejor comunicación, pero eso no es del todo acertado ya que si una empresa está brindando una gran cantidad de información y esta no es la correcta no están haciendo nada para que mejore el desempeño de sus empleados, pero si se brinda una información concreta, acertada y de calidad; el desempeño de los empleados se verá reflejado en toda la organización.

\section{Empowerment}

El empowerment se ha convertido en la filosofía necesaria para dirigir la organización del siglo XXI. La mayoría de las organizaciones tendrán que llevar a cabo una serie de rápidas transformaciones, deberán cambiar su estilo y manera en que se dirigen de acuerdo con los cambios de ambiente en los cuales tendrán que trabajar. (Wilson, 1996, p. 18).

El empowerment es un proceso con el cual se busca delegarle la autoridad y el poder a los empleados de una empresa, los empleados empiezan a asumir responsabilidad y de esta manera comienzan a participar en la toma de decisiones; esto se hace con el fin de obtener un mayor rendimiento de los empleados, también que se sienta dueño de su trabajo. Se trata de estimular a los empleados, de que tomen la iniciativa y riesgos, además de esto que se propongan metas para realizar su trabajo. Los empleados empiezan a tener el control.

\section{La motivación}

La palabra Motivación deriva del latín Motivus, que significa "causa del movimiento", el concepto motivación lo conforma la palabra motivo y acción, eso significa que para que un empleado se encuentre motivado y este comprometido con la empresa y rinda al $110 \%$ debe tener un motivo que lo lleve a la acción. 
Existen varios motivos posibles, como no sentirse bien remunerado, la mala relación con el superior, con otros compañeros o cliente, la falta de reconocimiento, la falta de desarrollo profesional, la rutina, los problemas personales, etc.

Para enfrentar a esta situación debemos volver a crear la ilusión del primer día en el empleado, volver a enamorarlo, entusiasmarlo y ayudarle a encontrar el motivo que lo lleve a la acción.

Una buena comunicación reforzará los pilares de la motivación del empleado, proporcionándole el motivo que necesita para pasar a la acción y permitiendo que estos recobren su entusiasmo y compromiso, elevando así las posibilidades del éxito de la empresa.

Pero, ¿cómo lograr empleados cien por ciento motivados y comprometidos, cuando en estudios como el realizado por el Sirota Survey Intelligence de Nueva York, el $85 \%$ de las empresas entrevistadas reveló una motivación y moral en sus empleados, profundamente descendente, después de los primeros seis meses de estancia?

La respuesta es fácil, aplicando el Endomarketing o marketing interno, un proceso de gestión -cíclico y continuo-que promueve la motivación de los trabajadores, el mejoramiento del clima organizacional y la obtención de un mayor grado de lealtad y compromiso en la relación empresa-trabajador. El endomarketing busca que los empleados se sientan parte de la empresa, que tomen conciencia de la importancia de sus aportes al fortalecimiento de la misma y que lleven a cabo acciones que redunden en un mayor beneficio y mejores resultados para la organización y para ellos mismos.

\section{Pregunta general}

¿Cuáles son las prácticas de marketing interno en los restaurantes de comidas rápidas FRISBY S.A en Pereira?

\section{Preguntas específicas}

¿Cómo se lleva a cabo el proceso de marketing interno en el restaurante de comidas rápidas FRISBY S.A?

¿Qué actividades de marketing interno se aplican en cada departamento?

¿Qué enfoque de marketing interno aplica Frisby S.A?

¿Qué énfasis se le hace al empleado según el cargo que ocupa en la organización?

\section{Diseño de la Investigación}

El método utilizado en la presente investigación es el cualitativo; pues, es necesario para la medición de variables no cuantificables como las percepciones de los colaboradores de la organización y su conducta. Sampieri (2003) refiere que el enfoque cualitativo se basa en métodos de recolección de datos sin medición numérica, como las descripciones y las observaciones. Por lo regular, las preguntas e hipótesis surgen como parte del proceso de investigación y éste es flexible, y se mueve entre los eventos y su interpretación, entre las respuestas y el desarrollo de la teoría. Su propósito consiste en "reconstruir" la realidad, tal y como la observan los actores de un sistema social previamente definido.

La metodología en una investigación cualitativa es inductiva, holística y fenomenológica. Es inductiva porque desarrolla conceptos partiendo de datos, holística, porque los datos, los escenarios y las personas no son reducidos a variables, sino considerados como un todo y siempre dentro de su marco teórico. (Soler, 2001, p.27).

\section{Técnicas e instrumentos}

Para el logro de los objetivos planteados se eligieron dos momentos y la aplicación de dos instrumentos para recolectar la información necesaria. 
El primer momento; entrevista en profundidad no estructurada, en la cual se vive una experiencia dentro de la organización, desde los diferentes departamentos para evidenciar los procesos internos. Heinemann (2003) define la observación participante como una forma de observación en la que el observador se introduce en el suceso; es decir, se realiza (mediante recolección de datos) en el campo no falsificado del suceso, el observador participa en el suceso y es considerado por los demás parte del campo de actuación.

El segundo momento; entrevista en profundidad no estructurada, con reiterados encuentros cara a cara entre investigador e informantes, encaminados hacia la comprensión de las perspectivas, experiencias o situaciones que tienen los colaboradores con sus propias palabras; el contenido, el número y secuencia de preguntas es determinado por el investigador, para obtener principalmente respuestas emocionales sobre las racionales.

\section{Resultados esperados}

A partir de este estudio se pretende ofrecer una pauta para diferentes organizaciones en cuanto a la importancia que tiene el autoanálisis corporativo, el análisis de los diferentes procesos de gestión en los momentos de comunicación e información de las compañías, la promoción y desarrollo del clima organizacional en coherencia con el valor de su marca y los objetivos institucionales, los procesos de motivación para el incremento del grado de lealtad y compromiso en la relación empresa cliente interno.

\section{Referencias}

Grönroos, C. (1994). "Marketing y gestión de servicios”. Madrid, España: Díaz de Santos S.A.

Alcaide, J, C. (2008). Fidelización de clientes. Madrid, España: ESIC EDITORIAL.

Robbins, S. (2005). "Administración. Octava edición. México: Pearson Educación”.

Grönroos, C. (1990). "Service management and marketing.Managing the moments of truth in service competition". Madrid, España: Díaz de Santos.

Wilson, T. (1996). "Manual del empowerment: cómo conseguir lo mejor de sus colaboradores". España: Ediciones gestión 2000.

Heinemann, k. (2003). "Introducción a la metodología de la investigación empírica". Barcelona: Editorial Paidotribo.

Hernandez, R. (2003). "Metodología de la investigación”. México, D. F: Mc Graw Hill. 